Réf. de l'article publié : Bessin M., «Conflits et ajustements professionnels dans la prise en charge sanitaire des détenus », in Cresson Geneviève, Druhle Marcel \& Schweyer François-Xavier, Coopérations, conflits et concurrences dans le système de santé, Rennes, éditions ENSP, 2003, chapitre 10, pp. 165-176.

\title{
Conflits et ajustements professionnels dans la prise en charge sanitaire des détenus
}

Marc Bessin, Chargé de recherche au CNRS, Centre d'Etude des Mouvements Sociaux, Ecole des Hautes Etudes en Sciences Sociales, Paris.

Quelles sortes de relations s'établissent entre professionnels et profanes lorsque les patients sont des prisonniers ? Dans le contexte d'une réforme de la santé en milieu carcéral, une enquête socio-ethnographique a permis d'observer de nouvelles relations professionnelles et des ajustements entre les soignants hospitaliers et les autres protagonistes de la relation de soins en milieu carcéral, particulièrement les détenus et les surveillants.

La loi de 1994 a accordé le bénéfice de la protection sociale à toutes les personnes incarcérées et a transféré leur prise en charge sanitaire de l'administration pénitentiaire au service public hospitalier. Concrètement, pour assurer les soins somatiques, chaque établissement de santé jumelé avec une prison devait créer une Unité de Consultations et de Soins Ambulatoires (UCSA). Ces services sont animés par une équipe pluridisciplinaire composée de personnels hospitaliers, mais les anciens soignants pénitentiaires peuvent aussi rejoindre la fonction publique hospitalière. Les structures psychiatriques (Service Médico-Psychologique régional : SMPR, qui relève d'un hôpital spécialisé) ont été renforcées par cette loi, dans une dynamique similaire.

La recherche sociologique (Bessin, Lechien, 2000) a consisté à conduire sur trois ans, dans deux prisons pour hommes, un travail de terrain, mêlant les perspectives ethnographiques de l'observation et de l'immersion relativement longue et répétée au sein des établissements afin de pouvoir accéder aux différents points de vue (détenus, soignants, agents et administration pénitentiaires, magistrats, travailleurs sociaux, etc.) ; la démarche ethnographique a été associée aux méthodes classiques de l'entretien, plus ou moins formalisé, avec ces acteurs. Nous avons observé et identifié, d'une part, les conditions et les représentations de la prise en charge sanitaire des détenus et, d'autre part, les pratiques professionnelles et les usages des prestations soignantes que cette prise en charge mettait en œuvre. Ce fut aussi l'occasion de porter un regard décentré sur la pénalisation du champ médico-social, la rationalisation gestionnaire de l'institution hospitalière et les tensions de l'humanisation relative de l'univers pénitentiaire. Nous nous sommes notamment intéressés aux effets de l'introduction, dans la gestion de la population pénale, de nouveaux intervenants extérieurs provenant du monde hospitalier et amenés, dans le conflit et l'ajustement, à négocier le partage de leurs attributs professionnels (déontologies, secrets, compétences, missions, lieux, temps...).

Le cadre problématique de cette recherche repose sur l'analyse des interactions et des concurrences d'humanité autour des relations soignantes. Dans la mesure où, en prison, quelle que soit sa mission première, chaque intervenant est enfermé dans une contradiction structurelle 
qui traverse le monde carcéral, pris entre la garde et l'entretien, l'interprétation par les acteurs de la réforme en termes de "choc des cultures" ne pouvait qu' appeler une recherche permettant de saisir les ajustements et les segmentations professionnels (Bucher, Strauss, 1961)1. L'observation de l'ordre négocié (Strauss, 1992) de ces relations a guidé notre démarche, à l'encontre du cadre interprétatif du "choc des cultures" entretenu par les professionnels de santé pour mettre en avant leur appartenance à une communauté médicale unifiée en tirant tous les avantages moraux d'une confrontation avec l'institution pénitentiaire ${ }^{2}$. Car si les soignants se présentent dans une neutralité affective ("soigner sans juger") et une intégrité déontologique ("chacun sa place"), nous avons observé des manières différenciées de partager les compétences avec les autres professionnels. Des recompositions de pratiques et des redéfinitions du travail et de l'éthique professionnelle se mettent en place dans l'ajustement des logiques pénitentiaires et sanitaires, chacun tentant de légitimer son travail, menacé par la dévalorisation liée au cadre d'exercice.

Ce texte insiste sur les formes de conflits inhérents à la situation des soignants en prison et réactivés par la réforme, et expose les modalités d'ajustements professionnels pour les dépasser. Il revient au préalable sur le contexte au sein duquel les relations professionnelles autour du soin s'établissent dans ce lieu d'exercice particulier que constitue le milieu carcéral.

\section{Un lieu d'exercice qui entrave la confiance dans les relations de soins}

L'amélioration objective de l'offre de soins n'implique pas mécaniquement un accès des détenus au statut de patients. Les conditions de la santé en prison et de l'accès aux prestations sanitaires, qui restent dérogatoires, favorisent une rhétorique du soupçon qui pèse sur la relation de soin et alimente les conflits professionnels.

L'accès aux services médicaux en prison reste fortement réglementé, soumis à une procédure de requête écrite et induisant une surjustification de la demande de soins. Si l'offre médicale est nettement renforcée avec la réforme, les UCSA demeurent en situation de monopole et ne permettent pas le libre choix du médecin. Des carences persistent dans certaines spécialités et les détenus sont astreints à de longues attentes. Les " extractions médicales " (transfert à l'hôpital de proximité) apparaissent comme un enjeu central entre les hôpitaux, les UCSA et les établissements pénitentiaires (enjeu du nombre et de la durée des hospitalisations, des personnels mobilisés, des risques pointés par l'administration pénitentiaire...). Les détenus, s'ils peuvent saisir une occasion de sortie de la prison, sont heurtés par les conditions dans lesquelles s'effectuent les extractions (transport, port humiliant de menottes et entraves) et les refusent parfois, surtout lorsqu'ils sont orientés vers l'hôpital pénitentiaire de Fresnes.

Au-delà, c'est surtout l'état de malade qui souligne le difficile accès des détenus au statut de patient. La convalescence est entravée, les régimes dérogatoires étant très limités. L'absence de droit du travail et de congés maladie oblige les détenus les plus pauvres à ne pas respecter les prescriptions médicales d'arrêt de travail. Pour ceux qui sont atteints de pathologies graves et stigmatisantes, comme le sida, l'ensemble des relations carcérales exerce une pression constante à la divulgation, ce qui illustre l'étroite marge de manœuvre d'un patient ordinaire en prison : le statut de malade ne s'impose pas par simple demande de soins, mais constitue un état variable

\footnotetext{
1 Pour une présentation française des travaux centrés sur les questions de segmentations professionnelle : (Dubar, Tripier, 1998). Pour une application au domaine médical : (Freidson,1984).

2 Pour un point de vue avisé représentatif - bien que nuancé - de cette posture médicale : (Obrecht, 1997).
} 
qui se justifie en permanence, sans quoi toutes les suspicions sont permises. Pour l'instant, aucun aménagement n'est prévu pour la prise en charge du handicap et de la dépendance, notamment des personnes âgées, les personnels pénitentiaires étant obligés d'improviser avec les moyens du bord.

Le sentiment d'illégitimité des prisonniers participe d'une défiance et d'une méfiance vis-à-vis des soignants. L'univers carcéral fonctionne largement au manichéisme : l'intervenant extérieur est sommé de "choisir son camp ", les soupçons de collusion avec l'administration pénitentiaire (participer aux objectifs sécuritaires) et de connivence avec les personnes libres pesant sur son travail. Les prisonniers intègrent l'idée selon laquelle ils ne peuvent accéder à des soins identiques à ceux qui sont administrés à l'extérieur. Cette illégitimité produit aussi l'illégitimité professionnelle à laquelle les soignants sont constamment renvoyés, tant par les patients que par leurs pairs hospitaliers. Les détenus perçoivent dans le regard des professionnels une mise à distance liée à leur condition d'hommes dépourvus de droits et condamnés moralement.

\section{L'hôpital incarcéré : de quelques ajustements professionnels}

Une fois ce contexte de méfiance et de défiance rapidement décrit, nous pouvons maintenant nous intéresser aux effets de l'arrivée des personnels hospitaliers, munis de leurs savoir-faire et de leurs certitudes, tentant d'importer une rationalisation des pratiques soignantes dans un univers qu'ils connaissent mal. Les contours des professions se redéfinissent dans une improvisation tout à la fois de coopérations et de protections, à travers le traitement ordinaire de la souffrance des détenus et de la violence carcérale. Violence qui redouble la précarité de destins sociaux dont le contrôle tend à contaminer les frontières entre la peine et le soin et à transformer les établissements pénitentiaires en "derniers" lieux de soins.

\section{“L'intrusion" de la rationalisation hospitalière}

Dans la plupart des établissements pénitentiaires, l'arrivée de nouveaux professionnels hospitaliers a été vécue comme une "intrusion" par les personnels de surveillance et, dans une moindre mesure, par les soignants relevant de l'administration pénitentiaire. Le terme "intrusion" souligne la violence de cette confrontation et la multiplication des situations conflictuelles enregistrées dans les premiers mois d'application de la réforme. Celle-ci a engagé une opération de reconversion des anciennes "infirmeries", totalement intégrées à l'ordre pénitentiaire. Mandatés pour la "création" de nouveaux services, les personnels hospitaliers ont importé leurs outils, leurs postures soignantes et leurs dispositions professionnelles. Ils ont imposé une délimitation physique des espaces sanitaires et pénitentiaires pour construire l'autonomie de leur intervention et substituer aux carences et à l'arbitraire de l'accès aux soins des détenus une forme d'égalisation médicale et de "responsabilisation" des patients.

Les conflits ont donc été produits par "l'intrusion" de nouveaux partenaires statutairement indépendants dans la gestion de la population pénale et par la logique même de la réforme, qui disqualifiait de fait les pratiques passées. Ils ont été principalement saisis à travers les tensions entre infirmières hospitalières et surveillants. Ces deux catégories professionnelles sont en effet les plus présentes, sont placées au bas de leur hiérarchie respective ${ }^{3}$ et sont les plus exposées aux

\footnotetext{
${ }^{3}$ En l'absence en prison d'aides soignantes.
} 
détenus et à la violence de l'ordre pénitentiaire. L'exemple des appels des surveillants des unités, appels pour des " urgences" traités par les infirmières des UCSA en maison d'arrêt, permet de saisir l'enjeu des conflits et la spécificité du travail soignant en prison : le rôle joué par les personnels pénitentiaires, partenaires profanes interposés entre soignants et détenus, mais aussi la gestion par les infirmières des demandes d'une population non sélectionnée, comme aux urgences hospitalières (Peneff, 2000 ; Bessin, 1997). Les conflits engagent donc la maîtrise, par chaque catégorie professionnelle, de ses conditions de travail et de la définition de ses missions ${ }^{4}$ : gestion de l'ordre précaire des unités pour les surveillants qui tentent de "se débarrasser" des prisonniers “ingérables" en obtenant leur passage à l'infirmerie, gestion de l'accès des détenus au statut même de patients pour les infirmières dont la charge de travail a été considérablement augmentée et renouvelée avec la réforme.

Dans les prisons étudiées, les tensions étaient redoublées par le genre des acteurs, par une série d'oppositions entre "féminité" et "virilité" professionnelles (sécurité/soins, répression/humanité, violence physique/écoute, détenus/patients, voyous/malades...). Placés sous un nouveau regard, les surveillants se sentaient renvoyés à l'indignité de leur métier face à des femmes représentant les missions nobles du soin et détentrices d'une autorité spécifique 5 . Autorité portant la marque que l'organisation hospitalière imprime aux comportements et aux gestes les plus anodins, jusqu'aux inflexions de la voix, autorité liée au partage du savoir médical, à la maîtrise de gestes touchant à l'intimité du corps et à la proximité avec la mort.

Ces conflits, aujourd'hui pacifiés mais ponctuellement remobilisés, permettent de saisir des enjeux plus implicites, à présent recouverts. Enjeux de l'humanisation relative de l'univers pénitentiaire et de ses contradictions. Tout d'abord, les surveillants, placés du côté de la sécurité et de la répression, peinent à défendre leur dignité professionnelle et à faire valoir, dans les conflits avec les infirmières, la part d'humanité de leur travail. Cette part d'humanité tend à être réduite par les transformations progressives des établissements pénitentiaires. L'amélioration des conditions d'existence des détenus est largement déléguée à des intervenants extérieurs, qui se chargent d'informer les prisonniers de leurs droits, de les soigner, de les écouter et de les occuper. La présence de ces intervenants, dont les missions s'ajoutent sans coordination à celles des surveillants, augmente mécaniquement les contrôles et limite les agents pénitentiaires à leur rôle sécuritaire, au moment même où ils sont incités à s'investir dans la "réinsertion " et sont confrontés à une population pénale dont les comportements les désarment (toxicomanes, délinquants sexuels, malades psychiatriques, jeunes sans avenir qui opposent conduites de défi...). Par ailleurs, les tensions entre infirmières et surveillants donnent à voir des opérations ordinaires de qualification, d'imputation et de gestion de situations qui ont trait à la violence carcérale. Face à un détenu violent et à bout, qui doit intervenir et contribuer à la pacification des unités ? Si les personnels pénitentiaires sont portés à assigner les nouveaux services médicaux au rôle de simples lieux de "décompression" pour les détenus, les soignants hospitaliers luttent pour imposer une hiérarchie proprement médicale des demandes.

Pour illustrer ces tensions, examinons rapidement le cas des toxicomanes en quête de produit, qui harcèlent les services médicaux pour soulager un état de manque. C'est une figure récurrente de la problématique de soignants en milieu pénitentiaire (essentiellement en maison d'arrêt), qui nourrit leurs suspicions plus ou moins généralisées sur la simulation des détenus et

\footnotetext{
${ }^{4}$ Pour une analyse détaillée de ces questions pour les agents pénitentiaires : (Chauvenet, Orlic, Benguigui, 1994).

${ }^{5}$ Nous avons développé cette question des valeurs et pratiques sexuées mobilisées dans la relation de soins en prison dans (Bessin, Lechien, 2002).
} 
«l'inauthenticité » de la relation. La rationalisation hospitalière a induit une modification des pratiques, consistant parfois à refuser des demandes de soins qui relevaient manifestement d'une recherche de substituts de psychotropes. Certains surveillants, surpris par cette rupture avec les habitudes antérieures qui privilégiaient l'apaisement au court terme du détenu toxicomane et de l'ensemble du bâtiment ("Ils ne veulent pas les soigner, alors qu'ils souffrent à en crever »), ont ensuite compris que les infirmières préféraient maintenir un protocole de soins qui s'avérait plus efficace dans la durée, y compris en terme d'ordre carcéral. Pour autant, cette ligne de conduite édictée par les soignants ne s'impose pas unilatéralement, l'existence de trafics, notamment de médicaments ou de produits de substitution, venant souvent troubler non seulement l'ordre carcéral, mais également la logique médicale. Les médicaments ne sont plus dilués dans des "fioles ", mais les médecins continuent de s'inquiéter sur les limites à donner aux prescriptions de psychotropes qui allègent les souffrances de l'emprisonnement. S'ils contribuent à " assommer" les détenus (la "camisole chimique"), les médicaments demeurent cependant une source de perturbation en prison par les risques de trafics et de suicide. Les prescripteurs peuvent ainsi devenir la cible de l'administration pénitentiaire, qui trouve par-là des responsables et un moyen commode de ne pas parler de dysfonctionnements plus profonds.

Au final, pénitentiaires et hospitaliers coopèrent, précisément parce qu'ils sont enfermés dans la même situation et s'affrontent à la même violence. Cette coopération peut même revaloriser le travail pénitentiaire d'observation. Avec l'importation de postures soignantes et d'outils hospitaliers, les nouveaux personnels importent de fait une plus grande vigilance à la santé, au corps, à l'hygiène, aux habitudes alimentaires et au suivi psychologique des détenus. Ils sont donc plus demandeurs d'informations sur l'existence quotidienne des prisonniers. De la qualité de l'observation des détenus dépendent la qualité des signalements aux services médicaux et la qualité du suivi sanitaire et psychologique. Les personnels des UCSA et des SMPR s'appuient sur le regard interposé des surveillants dans un système sanitaire où, à l'inverse de l'organisation hospitalière, les patients échappent largement au contrôle des soignants.

\section{Identités et éthiques professionnelles à l'épreuve des ajustements}

La rhétorique du "choc des cultures", qui prévalait à tous les niveaux d'application de la réforme, avait une fonction de protection permettant aux différents personnels de maintenir un voile sur l'enchevêtrement des logiques professionnelles en prison afin de préserver des certitudes quant aux valeurs morales assignées aux finalités de leurs missions (sécurité ou soin). Nous avons étudié ce cas typique de " drame social du travail " (Hughes, 1996) où chacun définit la situation selon des perspectives différentes, en nous concentrant sur les segmentations et différenciations, particulièrement lisibles aux frontières des interventions des agents et dans le partage de leurs compétences.

\section{Le secret médical à l'épreuve de la coopération professionnelle}

En premier lieu, la détention et la circulation de l'information, fondamentale en prison dans la mesure où elle conditionne la fonction de surveillance, donnent lieu à d'importantes négociations autour du secret médical. S'il est devenu emblématique des conflits initiaux autour de la réforme ${ }^{6}$, c'est qu'il symbolise les enjeux de la coopération professionnelle, au croisement des

\footnotetext{
${ }^{6}$ Une grande partie des arguments échangés au début de la réforme tournaient autour du secret médical, auquel les surveillants avaient l'habitude d'accéder. Ces conflits étaient régulièrement illustrés par l'élaboration de listes de
} 
visées à l'autonomie et des obligations à travailler ensemble. Par une conception absolue ou relative de la préservation du secret médical, s'observe une plus ou moins grande prise en compte des spécificités du milieu d'exercice. Au-delà des positions de principe, les ajustements s'effectuent dans la reconnaissance des compétences de chacun, le temps et la confrontation à certaines épreuves. Le secret pénal concernant les faits ayant entrainé l'incarcération met également à l'épreuve les personnels soignants, partagés entre leur déontologie et leur morale sociale, hésitant entre la banalisation de la condition pénale et son impossible neutralisation. Les soignants affirment ne pas vouloir connaître les faits, mais leur accès au travail d'écoute laisse ouverte la question. Selon la conception du travail relationnel, ce plus ou moins grand intérêt pour l'affaire renvoie aux liens qu'un soignant établit entre la peine et le soin.

\section{Les objectifs au principe de la coopération : entre ordre, peine et soin}

Certaines situations critiques (états de crise, risques de trafic ou de suicide par les médicaments) permettent d'observer les dilemmes posés aux soignants autour de leur participation à l'ordre carcéral. Les soins améliorent la situation des détenus, mais ne contribuent-ils pas ainsi à les apaiser et à leur faire accepter leur condition? L'instrumentalisation des infirmeries pénitentiaires aux fins de réduction des tensions carcérales était explicite avant la réforme, mais demeure aujourd'hui une source d'interrogations pour beaucoup d'hospitaliers, comme on a pu le voir à propos des toxicomanes. Pour autant, la souffrance d'un détenu en crise détermine leur intervention.

Mais les conflits de perspectives entre le soin et le maintien de l'ordre sont parfois redoublés par des conflits entre UCSA et SMPR, qui s'avèrent assez fréquents. Une situation observée dans une maison d'arrêt permet d'illustrer ce qu'un récent rapport de l'IGAS ${ }^{7}$ vient de souligner en évoquant par un bel euphémisme les "déficits de communication» et les relations "marquées par des logiques de territoire ». Suite à une bagarre au cours de laquelle une personne se blesse, le médecin de l'UCSA argue au téléphone de l'état d'agitation du patient et de son refus de se soigner. Un infirmier du SMPR se déplace mais observe que le détenu ne relève pas de son intervention, "l'urgence n'étant pas de rentrer en relation mais de recoudre sa boîte crânienne ». 18 heures sont entre temps passés et le chef de service de l'UCSA est parti : "Résultat, il a fallu appeler SOS médecins pour le recoudre ». Commentant cet épisode qui présente l'action de ses services à leur avantage, le directeur pénitentiaire profite de ces tensions exacerbées pour tenter une redistribution des valeurs morales («Au final, c'est nous qui avons réglé le problème »). Dans ces conflits récurrents, l'administration est le plus souvent portée à adopter le point de vue des psychiatres, plus enclins à prendre en compte le contexte et les contraintes de l'institution, et à déplorer la tradition organiciste des somaticiens hospitaliers, perçus comme appréhendant le patient à partir des seules déficiences de ses fonctions organiques en éludant l'histoire et les conditions d'existence des détenus.

\section{Le partage du " sale boulot " ou la coopération des détenus}

À travers l'exemple de quelques situations de confrontation avec le corps malade des détenus, se pose enfin la question de la gestion des hommes incarcérés les plus diminués et du partage du

malades séropositifs, pratique qui était courante avant la réforme et qui est encore régulièrement réclamée par certains agents pénitentiaires.

${ }^{7}$ Le Monde, 18 avril 2002. 
"sale boulot" ("dirty work" (Hughes, 1996)). Qui doit s'occuper des handicapés, secourir les grabataires, assister les toxicomanes les plus dépendants ? Infirmières et surveillants luttent pour ne pas ajouter cette charge à leur travail, en l'absence d'aides-soignantes ${ }^{8}$. Consacrant une part importante de leur temps à des tâches administratives et techniques dont elles sont déchargées à l'hôpital, catégorie professionnelle la plus présente et la plus accessible, les infirmières détiennent de fait le monopole des relations affectives avec les détenus, à travers les consultations et les soins. Se protégeant, elles sont donc portées à déléguer l'assistance aux handicapés, habituellement assurée par des aides-soignantes. De leur côté, les surveillants, tentant de construire des barrières de distinction entre les détenus et eux, sont également déstabilisés par les prisonniers malades qui suscitent plus la compassion que la méfiance. Ils redoutent ainsi un glissement du relationnel à "l'affectif" (Drulhe, 2000) qui paralyse les rapports sécuritaires, même si tout ce qui "humanise" et singularise les détenus peut aussi fonctionner comme un mode de prévention des violences.

Nous avons pu assister à une scène particulièrement révélatrice des embarras induits par la présence d'un handicapé défiant toute l'organisation matérielle de la prison. L'accueil d'un homme en fauteuil transféré s'effectue au seuil de la cellule dont la porte est trop étroite. Le groupe de responsables mesure toutes les adaptatations matérielles que l'ouvrier doit effectuer en urgence, puis on fait le point sur l'aide à apporter plusieurs fois par jour à cette personne complètement dépendante. Le petit groupe se tourne alors vers l'infirmière de l'UCSA, qui fait alors savoir que ces soins ne relèvent pas de sa compétence. Même si aucun crédit n'est prévu pour ce type de détenus, le directeur de l'établissement ouvrira finalement une ligne budgétaire pour faire appel à un service d'aide à domicile. En attendant sa mise en place, ce sont des codétenus qui ont permis à cet homme de manger, se coucher et se laver. La prise en charge des prisonniers les plus dépendants revient ainsi largement aux codétenus. Or les hommes les plus "dévirilisés" dont il faut assurer la toilette suscitent un même dégoût ou une même pitié. Ceux qui sont atteints de maladies incurables ou de troubles psychiatriques incarnent surtout, aux yeux des prisonniers, l'abandon de soi et parfois la mort, spectacle insupportable du dépérissement ou de la "victoire " du système pénitentiaire, avec la destruction des détenus transformés en "fous " ou en "épaves". Ainsi, l'accompagnement des plus malades peut fonctionner comme un système de délégations en cascade, où chacun travaille à la préservation d'une distance avec une population qui symbolise soit l'exposition au risque de dévalorisation professionnelle, soit l'exposition au risque de la mort carcérale.

Mais dans cette répartition des tâches les moins nobles, les détenus occupent la position la plus fragile. Aucune déontologie professionnelle ne définit leur place dans le système sanitaire carcéral. Ils peuvent donc apporter leur concours aux malades incarcérés ou les mettre à l'écart et les " punir" de leurs défaillances. De fait, ils sont indirectement mais souvent sollicités dans la prise en charge de la santé : aide à la rédaction de courriers pour les UCSA et les SMPR en maison d'arrêt, soutien matériel et parfois affectif aux malades en convalescence, signalements, engagement dans des groupes de parole autour de l'alcoolisme... Cette participation multiforme des détenus constitue un levier pour l'administration pénitentiaire : dans la répartition stratégique de la population pénale et l'affectation des cellules, le choix des codétenus, des "voisins" ou d'un "doublage" est pesé. Certains prisonniers tentent de faire reconnaître cette participation, comme un " ancien " qui revendique l'instauration d'un " bénévolat sanitaire" en prison, à partir de son expérience de soutien quotidien à de jeunes codétenus toxicomanes placés dans un état

\footnotetext{
${ }^{8}$ C'est à cette catégorie de personnel qu'incombe le "sale boulot" à l'hôpital, incluant le travail invisible dédié aux "relations" avec les patients : (Arborio, 2001).
} 
d'abandon extrême. Cette revendication pose la question de l'autonomie et de la dignité des détenus, du statut des pratiques d'entraide.

$* * * * *$

Les représentations des soins en prison et les présentations de soi des professionnels médicaux et soignants ${ }^{9}$ n'en épuisent pas les analyses. La perspective ethnographique adoptée dans cette recherche a privilégié les pratiques et les dilemmes auxquelles elles mènent, en situation. Ce lieu d'exercice traversé par le manichéisme et le soupçon induit en effet, paradoxalement, tous ceux qui y sont confrontés à coopérer, parfois dans le conflit, souvent dans la concurrence, en mettant tous les jours à l'épreuve la définition propre qu'ils se font de leur mission. Cette perspective élargit aussi l'analyse aux pratiques profanes des détenus dans ce système de la santé incarcérée. Il faudrait prolonger cette présentation de quelques ajustements professionnels autour des soins en prison par les questions de légitimation professionnelle induite par la menace permanente, lorsqu'il s'agit de lieux et de populations stigmatisés comme la prison et les détenus, de dévalorisation liée à des effets d'homologie sociale. On intégrerait alors dans le système de coopération professionnelle induite par les soins en prison, les collègues hospitaliers des personnels de l'UCSA, ceux des services d'urgence ou d'hospitalisation des hôpitaux au sein desquels les détenus sont quelquefois amenés à être soignés. D’autres types de conflits pourraient dès lors être décrits.

\section{Bibliographie}

Arborio A.-M. (2001) Un personnel invisible. Les aides soignantes à l'hôpital, Paris, Anthropos. Bessin M., Lechien M.-H. (2002), "Hommes détenus et femmes soignantes : l'intimité de la relation de soins en prison ", Ethnologie Française, "Intimités sous surveillance", vol. XXXII, 1, pp. 69-80.

Bessin M., Lechien M.-H. (2000), Soignants et malades incarcérés : conditions, pratiques et usages des soins en prison, CEMS, CES, EHESS, 415 pages.

Bessin M., (1995), "Le social aux urgences hospitalières : les enjeux d'une restructuration ", Les cahiers de la sécurité intérieure, $\mathrm{N}^{\circ} 22$, «Les métiers de l'urgence », 4ème trimestre, pp. 57-65.

Bucher R., Strauss A. (1961), "Professions in process", American Journal of Sociology, 66, (4), pp. 325-334, trad. fr. «La dynamique des professions » in Strauss, 1992, pp. 67-86.

Chauvenet A., Orlic F., Benguigui G. (1994), Le monde des surveillants de prison, Paris, PUF.

Drulhe M. (2000), "Le travail émotionnel dans la relation soignante professionnelle. Un point de vue au carrefour du travail infirmier", in Cresson G., Schweyer F.-X., Professions et institutions de santé face à l'organisation du travail. Aspects sociologiques, Rennes, Editions ENSP, pp. 1529.

Dubar C., Tripier P. (1998), Sociologie des professions, Paris, Armand Colin, Coll. U.

Freidson E. (1984), La profession médicale, trad. fr., Paris, Payot (1re ed. américaine 1970 sous le titre Profession of Medicine).

Hughes E.-C. (1996), Le regard sociologique. Essais choisis, Paris, Editions de l'Ecole des hautes études en sciences sociales.

Le Caisne L. (2000), Prison. Une ethnologue en centrale, Paris, Odile Jacob.

Milly B. (2001), Soigner en prison, Paris, PUF.

Obrecht O. (1997). Une réforme au milieu du gué, Revue française des affaires sociales, 51, 1, pp.117-129.

\footnotetext{
${ }^{9}$ Pour une telle posture de recherche : (Milly, 2001).
} 
Peneff J. (2000), Les malades des urgences. Une forme de consommation médicale, Paris, Métailié.

Rostaing C. (1997), La relation carcérale. Identités et rapports sociaux dans les prisons de femmes, Paris, PUF, Coll. Le lien social.

Strauss A. (1992), La trame de la négociation, sociologie qualitative et interactionnisme, Textes réunis et présentés par I.Baszanger, Paris, L'Harmattan. 